\title{
Non-progressive leukoencephalopathy with bilateral temporal cysts
}

\author{
ANTÓNIO LEVY GOMES, ${ }^{1}$ JOSÉ PEDRO VIEIRA, ${ }^{2}$ JOANA SALDANHA ${ }^{3}$ \\ ${ }^{1}$ Department of Pediatrics, Hospital de Santa Maria, Lisboa, Portugal; ${ }^{2}$ Department of Pediatrics, Hospital Fernando \\ Fonseca, Amadora, Portugal; ${ }^{3}$ Department of Pediatrics, Hospital Fernando Fonseca, Amadora, Portugal.
}

We report two cases of a peculiar leukoencephalopathy with temporal cysts. Both patients have a non-progressive neurological disorder with mental retardation, microcephaly and sensorineural deafness although clinical differences between them may reflect a different aetiology.

The metabolic disorders with white matter involvement and the recently described leukoencephalopathies (Van Der Knaap disease, 'vanishing white matter disease') were excluded based on clinical, biologic and imaging findings. Cytomegalovirus infection is a likely possibility in the first case although the magnetic resonance imaging picture is only partially similar to previously reported cases.

Our patients are strikingly similar to the patients reported by Deonna et al. and Olivier et al. We discuss the clinical and imaging findings in our patients and the differential diagnosis considering the known disorders of the white matter in childhood.

Keywords: Leukoencephalopathy. Temporal cysts. Magnetic resonance imaging.

\section{Introduction}

Advances in the understanding of white matter diseases in children were made possible with the introduction of magnetic resonance imaging (MRI) and magnetic resonance spectroscopy (MRS) into clinical practice. Previously known white matter diseases with metabolic defect were found to have a relatively specific pattern that could help in differential diagnosis. Two new diseases were recognized by clinical criteria and appearance on MRI.1,2

Some cases of leukoencephalopathy in children still remain undiagnosed. We present the clinical history and investigations of two patients with a peculiar leukoencephalopathy with temporal cysts. These patients have an apparently static disorder, clinically different in terms of severity and have microcephaly and sensorineural deafness in common.

\section{Case reports}

\section{Patient 1}

Patient 1 is a 24-month-old female. She is the only child of healthy, unrelated parents. Gestation was normal until the 37 th week when some reduction of amniotic fluid was noted and the fetal weight was estimated to be at the 10th centile. Delivery was by caesarean section at 37 weeks. The Apgar scores were 9 and 10 ( 1 and $5 \mathrm{~min}$ ). Birthweight was $1930 \mathrm{~g}$, length $45 \mathrm{~cm}$ and head circumference $30 \mathrm{~cm}$. The newborn was clinically well with normal results of physical and neurological examination. Because of low birthweight, toxoplasmosis, rubella, cytomegalovirus and herpes simplex (TORCH) screen was performed and the results were consistent with congenital cytomegalovirus (CMV) infection: positive IgM, IgG and CMV 
antigenuria. Brain ultrasound described slight enlargement of the lateral ventricles more on the left and a subependimal cyst on the left. Results of ophthalmologic examination and liver function tests were normal.

At follow-up, psychomotor development was globally delayed. The girl sat alone at 12 months and walked without support at 21 months. She has normal visual contact, recognizes family members and waves goodbye. Vocalization is limited to a few monosyllabic sounds. She is hypotonic with normal reflexes and has no signs of ataxia or cranial nerve abnormalities except for lack of response to sounds. Audiologic evaluation has disclosed a profound bilateral sensorineural hearing loss. Head circumference is now $43 \mathrm{~cm}$, and remains below the 5th centile. Weight and length are in the 5th centile.

Brain MRI, performed when she was 11 months revealed a leukoencephalopathy involving the temporal, parietal and occipital lobes with a grossly symmetrical pattern. In the temporal lobe a cystic change is striking with signal evolution similar to cerebrospinal fluid (CSF) (Figs 1 and 2).

Metabolic studies (amino acids, organic acids, blood and CSF lactate and pyruvate, ammonia, arylsulphatase, galactocerebrosidase, very long chain fatty acids) were normal. The polymerase chain reaction (PCR) for CMV was negative in the CSF. Creatine kinase was normal.

\section{Patient 2}

Patient 2 is a 6-year-old boy, the second child of healthy, non-consanguineous parents. No relevant perinatal problems were documented. Birthweight was $3545 \mathrm{~g}$ and head circumference was $33 \mathrm{~cm}$. He was referred for neurological evaluation when he was 4 months because of hypertonia. He had good visual contact and appeared interested in his surroundings. Neurological examination was normal except for exaggerated myotactic reflexes. Two months later he was re-evaluated and tone in the extensor axial muscles was definitely increased. Abnormal response to sound was present. The brainstem auditory evoked responses (BAERs) confirmed profound bilateral sensorineural loss. On follow-up the patient had a progressive but very slow increase of motor ability; he walked with support at 4 years and is now able to take some steps without support. Voluntary prehension was present at 2 years. He vocalizes only a few sounds but does use some non-verbal communication.
The neurological examination reveals bilateral, symmetric pyramidal signs without other abnormalities. Head circumference is $48 \mathrm{~cm}$. He has a happy disposition and sometimes displays paroxysmal laughter which combined with a slightly dysmorphic

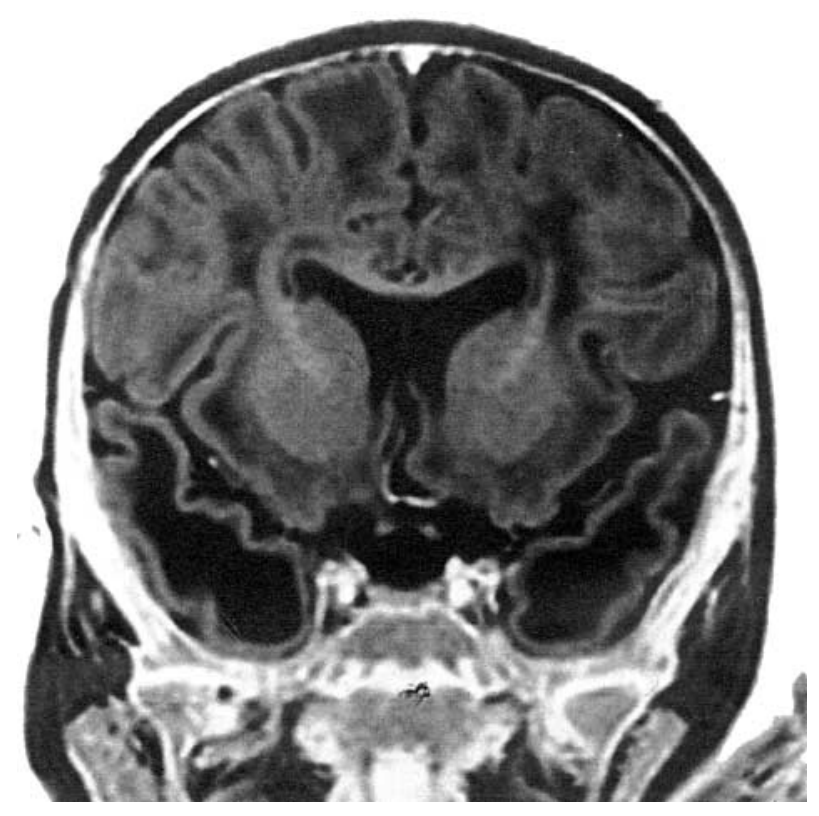

Fig. 1. MRI (T1 weighted) from patient 1 showing the temporal cysts.

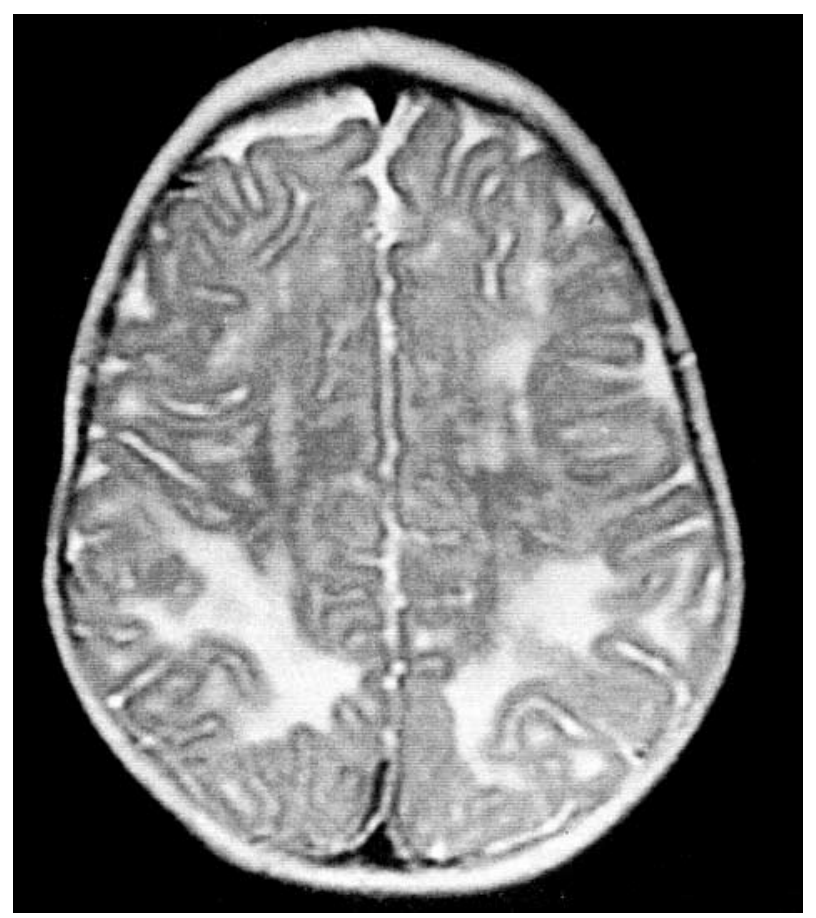

Fig. 2. MRI (T2 weighted) from patient 1 showing the posteriorly located areas of abnormal hypersignal in the white matter. Oedema of the gyri is not present. 
face prompted us to demand a chromosomal study for Angelman's syndrome which was negative. Ophthalmologic examination was normal.

Metabolic studies (amino acids, organic acids), TORCH studies, and electromyogram (EMG) were normal.

Brain MRI showed a pattern very similar to that described for patient 1: a symmetrical leukoencephalopathy with temporal cysts (Figs 3 and 4).

This study was repeated 1 year after the first study: the pattern of lesions remained unchanged.

\section{Discussion}

We report our observation of two children with a non-progressive encephalopathy with delayed development, microcephaly and deafness. The MRI showed bilateral temporal cysts and a leukoencephalopathy, negative results concerning a metabolic aetiology and, in the first case, laboratory evidence of congenital CMV infection.

Our patients did not have any clinical evidence of systemic disease in the neonatal period which could be expected with late CMV infection. Both behave clinically as if they had static encephalopathy although patient 2 is more severely handicapped in his cognitive and motor abilities.

Patient 1 had a documented congenital CMV infection that could cause microcephaly, retarded psychomotor development, and sensorineural deafness. The ophthalmologic examination showed no sign of congenital ocular CMV infection and the CSF examination was normal; the PCR for CMV was negative. Her neonatal brain ultrasound was reported as having a non-specific slight enlargment of the ventricular system and could not detect the temporal cysts; we do not know if this is the result of insufficient resolution or of progressive central nervous system disease.

In approximately $1.4 \%$ of asymptomatic neonates CMV can be isolated from the urine. ${ }^{3}$ Of all prenatally infected children (characterized by the presence of virus in tissues and urine) only $19 \%$ are symptomatic at birth. ${ }^{3}$ In the study by Fowler and co-workers ${ }^{4}$ hearing loss (either already present at birth or delayed in onset) and microcephaly (sometimes progressive) were two important features of congenital CMV infection. This infection could also be responsible for milder neurological manifestations including mental retardation and specific language disorders.

In CMV congenital infection MRI studies can be normal or have several abnormal findings. ${ }^{5-7}$

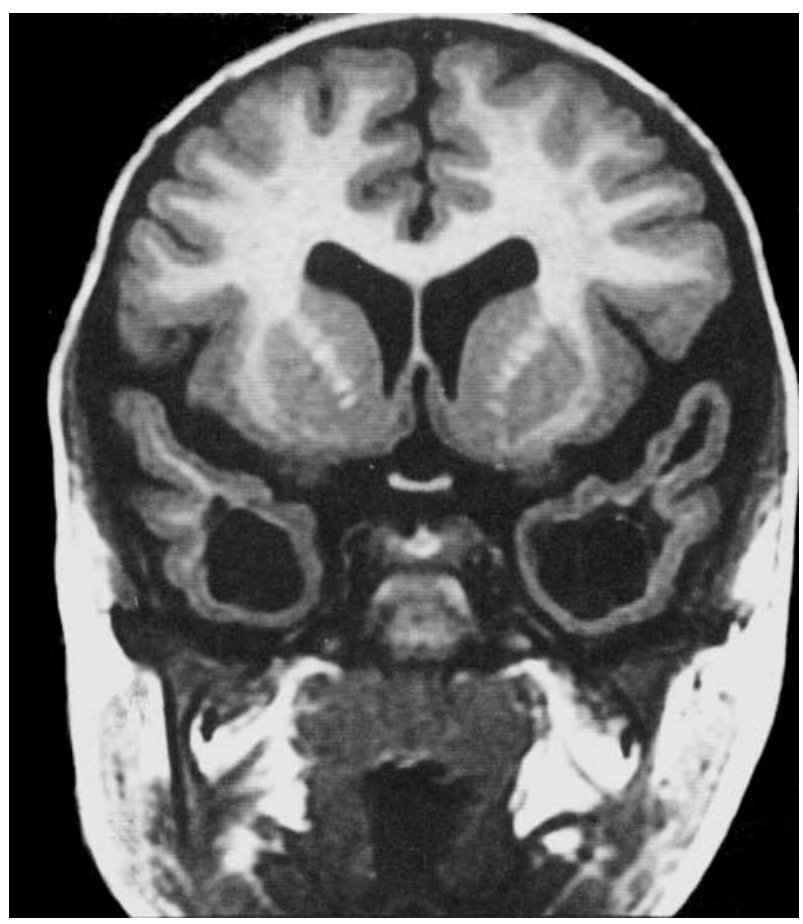

Fig. 3. MRI (T1 weighted) from patient 2 showing the temporal cysts.

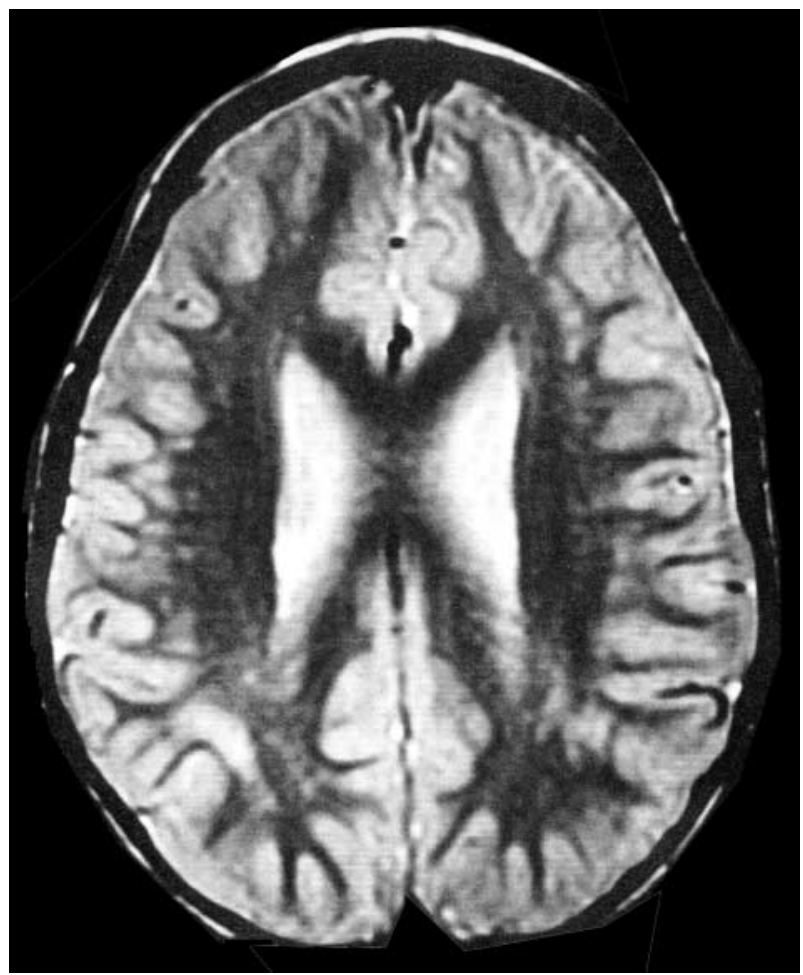

Fig. 4. MRI (T2 weighted) from patient 2. Occipital white matter also displays hypersignal. 
Barkovich et al. ${ }^{7}$ described ten patients with congenital CMV infection and several MRI abnormalities: cerebellar hypoplasia, disorders of neuronal migration and white matter abnormalities including haemorrhage and calcification. Four patients had anterior temporal lobe cysts. The authors regarded this finding as a result of late (third trimester) infection at a time when neuronal migration and cortical plate formation had already occurred resulting in a more selective lesion of glial cells (including the oligodendroglia). They also pointed out that this finding could be non-specific for this infection. Barkovich's patients were also different from our patient 1 because they had associated disorders of neuronal migration and/or of white matter signal suggesting haemorrhage or calcification. To our knowledge no other reports of temporal cysts exist in congenital CMV infection.

Our investigations for known metabolic leukoencephalopathies were negative, including the leukodystrophies (metachromatic and Krabbe's), organic acidurias, mitochondrial diseases, and Canavan's disease. Alexander's disease which has no known metabolic marker is, however, reasonably excluded considering the clinical picture and the MRI findings. ${ }^{8}$ Our patients do not have the clinical picture of congenital myopathy, the creatine kinase is normal in patient 1 and the MRI findings are also different from those described in the congenital muscular dystrophies.

Van der Knaap et al. ${ }^{1}$ described several patients with a diffuse leukoencephalopathy with swelling, temporal and frontoparietal cysts, and a mild clinical course. These children had normal or near-normal early development with late-onset ataxia, but they uniformly presented in the first year of life with progressive macrocephaly., ${ }^{1,9-11}$ Our patients also have temporal cysts but differ from van der Knaap's patients in several aspects namely the more restricted white matter disease with predominant involvement of the parietooccipital areas, no swelling of the gyri, the microcephaly and the apparently static clinical course.

Vanishing white matter disease is also markedly different from what was seen in our patients because this disorder has a more extensive white matter involvement, a clinical course with progressive and acute deterioration, and the cystic changes have no predilection for the temporal lobe. ${ }^{2}$

Olivier and co-workers ${ }^{\mathbf{1 2}}$ reported three patients with an MRI picture very similar to that shown in our cases. They were described as non-dysmorphic, spastic and severely delayed. One was microcephalic. None had deafness. Two patients were siblings raising the question of a hereditary autosomal recessive disorder.
Our cases are also remarkably similar in MRI appearance to three patients reported by Deonna et al.;13 their clinical course was, however, variable and considerably different in terms of severity (one was described as having spastic-dystonic diplegia with normal cognitive development, another as having global developmental delay and epilepsy, the third as having motor delay and hypotonia). These children also had an apparently static encephalopathy.

We think therefore that neither of our patients has one of the known white matter disorders and both present a peculiar MRI pattern which, considering the clinical picture, could be the result of problems of brain development with a special affinity to the white matter. Congenital CMV infection could be the aetiology for some cases.

\section{References}

1 van der Knaap M S, Barth P G, Stroink $H$ et al. Leukoencephalopathy with swelling and a discrepantly mild clinical course in eight children. Ann Neurol 1995; 37: 324-334.

2 van der Knaap M S, Barth P G, Gabreels F J M et al. A new leukoencephalopathy with vanishing white matter. Neurology 1997; 48: 845-855.

3 Stagno S. Cytomegalovirus. In: Remington J S, Klein J O (eds) Infectious Diseases of the Fetus and Newborn, 4th edn. Philadelphia: W B Saunders Co, 1995: 312-354.

4 Fowler $\mathrm{K}$ B et al. The outcome of congenital cytomegalovirus infection in relation to maternal antibody status. New Engl J Med 1992; 326: 663-667.

5 Boesch C, Issakainen J, Kewitz G et al. Magnetic resonance imaging of the brain in congenital cytomegalovirus infection. Pediatr Radiol 1989; 19: 91-93.

6 Steinlin M I, Nadal D, Eich G F et al. Late intrauterine cytomegalovirus infection. Pediatr Neurol 1996: 249-253.

7 Barkovich A J, Lindan C E. Congenital cytomegalovirus infection of the brain: Imaging analysis and embryologic considerations. Am J Neuroradiol 1994; 15: 703-715.

8 Pridmore C L, Baraitser M, Harding B et al. Alexander's disease: clues to diagnosis. J Child Neurol 1993; 8: 134-144.

9 Mejaski-Bosnjak V, Besenski N. Megalencephalic leukoencephalopathy: a further case of a new neurodegenerative white matter disease. Dev Med Child Neurol 1997; 39: 561-563.

10 Goutières F, Boulloche J, Bourgeois M, Aicardi J. Leukoencephalopathy, megalencephaly, and mild clinical course. A recently individualized familial leukodystrophy. Report on five new cases. J Child Neurol 1996; 11: 439-444.

11 van der Knaap M S, Valk J, Barth P G et al. Leukoencephalopathy with swelling in children 
and adolescents: MRI patterns and differential diagnosis. Neuroradiology 1995; 37: 679-686.

12 Olivier M, Lenard H G, Aksu F, Gartner J. A new leukoencephalopathy with bilateral anterior temporal lobe cysts. Neuropediatrics 1998; 29: 225-228.
13 Deonna $\mathrm{T}$, Roulet E, Vallée $\mathrm{L}$ et al. Bilateral temporal cysts, focal leukodystrophy and nonprogressive encephalopathy in three children: a special entity? Dev Med Child Neurol 1998 (Supp 79); 40: 18. 